\title{
Lupus anticoagulant and mortality in patients hospitalized for COVID-19
}

\author{
Carmine Gazzaruso ${ }^{1,2}$ (1) $\cdot$ Giuseppe Mariani $^{3} \cdot$ Carolina Ravetto $^{3} \cdot$ Laura Malinverni $^{3} \cdot$ Elena Tondelli $^{3}$. \\ Maria Cerrone ${ }^{1} \cdot$ Vittorio Sala ${ }^{3} \cdot$ Luigi Bevilacqua $^{3} \cdot$ Teodoro Altavilla $^{4} \cdot$ Adriana Coppola $^{2} \cdot$ Pietro Gallotti $^{3}$
}

Accepted: 3 November 2020 / Published online: 7 November 2020

(c) Springer Science+Business Media, LLC, part of Springer Nature 2020

\begin{abstract}
Coronavirus disease 2019 (COVID-19) is characterized by a procoagulant state that can lead to fatal thromboembolic events. Several studies have documented a high prevalence of lupus anticoagulant that may at least partially explain the procoagulant profile of COVID-19. However, the association between lupus anticoagulant and thrombotic complications in COVID-19 is controversial and no study has specifically evaluated the impact of lupus anticoagulant on mortality. The aim of our study was to investigate the association between lupus anticoagulant and mortality in a large group of 192 consecutive patients hospitalized for COVID-19. Lupus anticoagulant was found in 95 patients (49.5\%). No difference in the percentage of patients with lupus anticoagulant was observed between 130 survivors and 62 non-survivors $(47.7$ versus $53,2 \% ; \mathrm{p}=0.4745)$. When the combined outcome of death or need for mechanical ventilation in survivors was taken into account, the difference in the prevalence of patients with lupus anticoagulant between the patients with the combined outcome $(n=76)$ and survivors who did not require mechanical ventilation $(n=116)$ was not significant $(52.6 \%$ versus $47.4 \%$; $\mathrm{p}=0.4806)$. In multivariate analysis predictors of mortality or need for mechanical ventilation in survivors were obesity, low oxygen saturation and elevated troponin levels measured on admission. In conclusion, our study did not show any association of lupus anticoagulant with mortality and with need for mechanical ventilation in survivors. The role of obesity, low $\mathrm{SaO} 2$ and elevated troponin levels as predictors of a worse prognosis in patients hospitalized for COVID-19 was confirmed.
\end{abstract}

Keywords COVID-19 $\cdot$ thrombosis $\cdot$ lupus anticoagulant $\cdot$ death $\cdot$ Intensive care

\begin{tabular}{|c|c|}
\hline \multicolumn{2}{|c|}{ Abbreviations } \\
\hline BMI & Body mass index \\
\hline $95 \% \mathrm{CI}$ & $95 \%$ confidence intervals \\
\hline COVID-19 & Coronavirus disease 2019 \\
\hline OR & Odds ratio \\
\hline SARS-CoV2 & $\begin{array}{l}\text { Severe Acute Respiratory Syndrome-Coro- } \\
\text { navirus } 2\end{array}$ \\
\hline
\end{tabular}

Carmine Gazzaruso

c.gazzaruso@gmail.com

1 Emergency Unit and Endocrinology Unit, Istituto Clinico "Beato Matteo" (Hospital Group San Donato), Corso Pavia, 84, 27029 Vigevano, Italy

2 Centre for Applied Clinical Research (Ce.R.C.A.), Istituto Clinico "Beato Matteo" (Hospital Group San Donato), Corso Pavia, 84, 27029 Vigevano, Italy

3 Internal Medicine Unit, Istituto Clinico "Beato Matteo" (Hospital Group San Donato), Vigevano, Italy

4 Neurology Unit, Istituto Clinico "Beato Matteo" (Hospital Group San Donato), Vigevano, Italy

\section{Highlights}

- COVID-19 is characterized by a procoagulant state.

- The thromboembolic risk in patients with COVID-19 is high.

- Lupus anticoagulant is common in patients with COVID19.

- Lupus anticoagulant does not predict mortality in COVID-19.

- Lupus anticoagulant does not predict need for mechanical ventilation in COVID-19.

\section{Introduction}

About $15-20 \%$ of the patients with Coronavirus Disease 19 (COVID-19) experience a severe clinical course characterized by an interstitial pneumonia that often requires hospitalization [1]. A large proportion of these patients can 
have a very high arterial and venous thromboembolic risk [2]. Indeed, they can develop venous thromboembolism, pulmonary embolism, disseminated intravascular coagulation, acute respiratory distress syndrome, stroke, myocardial ischemia that can lead to death in about one third of the patients requiring hospitalization [2, 3]. However, microthrombi were found within small lung arteries of all the COVID-19 patients who died [4]. The high risk for thromboembolism is attributed to a procoagulant state due to an excessive inflammatory response to the viral infection [2,3]. Many abnormal coagulation parameters, including D-dimer, fibrinogen degradation products, antithrombin, fibrinogen, activated partial-thromboplastin time, prothrombin time, have been found in patients hospitalized for COVID-19 and they may be associated with worse outcomes, including death [5-9]. Some studies documented the presence of antiphospholipid antibodies [7, 10] and therefore their role in increasing the thrombotic risk of COVID-19 was hypothesized. However, the prevalence of antiphospholipid antibodies (anticardiolipin and anti-beta2-glycoprotein) was very low in patients with COVID-19 [7, 10-12,14-16] and their association to thromboembolic events was not found in specific studies [11-13]. On the contrary, several studies found that the prevalence of lupus anticoagulant was very high in patients with severe COVID-19 [14, 17-19]. However, at the moment it is unclear whether the presence of lupus anticoagulant is expression or causation of the hypercoagulable state described in COVID-19 [14, 17-19]. In addition, data on the prognostic usefulness of lupus anticoagulant are scarce and conflicting. A recent study found an association between lupus anticoagulant and thrombotic events in a small group of patients with COVID-19 [20], but another study did not find any association between lupus anticoagulant and thrombotic complications in critically ill COVID-19 patients [13]. However, no study specifically analyzed whether lupus anticoagulant can be used as a predictor of worse outcomes, in particular of mortality, in patients with severe COVID-19. The aim of our study was to investigate the possible association between the presence of lupus anticoagulant and mortality in a large group of patients hospitalized for COVID-19.

\section{Materials and methods}

For this study 192 consecutive patients admitted to our hospital from 15 to 2020 to 30 April 2020 for pneumonia due to Severe Acute Respiratory Syndrome-Coronavirus 2 (SARS-CoV2) have been retrospectively evaluated. Pneumonia was documented by Computed Tomography of the chest and SARS-CoV2 infection was confirmed by Real Time-Polymerase Chain Reaction. They were followed from admission to discharge. On admission, the presence of lupus anticoagulant was established together with all the other laboratory tests routinely made in patients hospitalized for COVID-19. Lupus anticoagulant analysis was exploited according to the recommendations of the International Society on Thrombosis and Haemostasis [21]. Prothrombin time and activated partial thromboplastin time were measured by commercial kits (Werfen, Barcelona, Spain). The patients were treated for COVID-19 according to the protocol of our hospital: hydroxychloroquine, lopinavir/ritonavir, antibiotics (doxiciclin and ceftriaxone) and a prophylactic dose of enoxaparin. Other specific treatments, including tocilizumab, therapeutic anticoagulation, steroids, antithrombin concentrate, were used on the basis of clinical course of each patient. For each patient the change from prophylactic to therapeutic anticoagulation was decided on a clinical basis: high D-dimer on admission, important increase of D-dimer during hospitalization, any clinical, laboratory or imaging suspicion of thrombotic complication, worsening clinical progression and respiratory failure were indications for the change. Lupus anticoagulant was not taken into account as its clinical significance in COVID-19 was still uncertain. It was used to stratify the individual risk of future thrombotic risk. Five patients were already on therapeutic anticoagulation prior to admission ( 3 on direct oral anticoagulants and 2 on warfarin) and were continued on it. The study was carried out according to the ethical standards of the institutional research committee and the 1964 Helsinki declaration.

In this retrospective study the primary outcome was death. A combined outcome of death or need for mechanical ventilation in survivors was also taken into account.

Statistical analysis. To find differences between two groups the Student t-test was used, while the Pearson Chisquared was carried out for frequency comparisons. Nonnormally distributed variables were log-transformed before each analysis. Survival curves were estimated by the KaplanMeier test and compared by the Mantel log-rank test. The effect of the variables with a $p<0.1$ at the univariate analysis on the primary outcome was evaluated in a multivariate Cox regression analysis. In addition, the effect of the variables with a $\mathrm{p}<0.1$ at the univariate analysis on the presence of lupus anticoagulant was evaluated in a multivariate logistic regression analysis. Each variable was dichotomized before the analysis. A p $<0.05$ was considered statistically significant.

\section{Results}

Table 1 depicts the features of the whole study population and of the patients stratified in survivors and non-survivors during the follow-up period (18.8 \pm 13.2 days - range 1-79). As shown, mean age, percentage of patients with lung disease, BMI, CRP, D-dimer, troponin and lactase dehydrogenase were significantly greater, and $\mathrm{SaO} 2$ significantly 
lower in non survivors than in survivors. The proportion of patients treated with therapeutic anticoagulation was significantly greater in non-survivors than in survivors. Among the five patients on therapeutic anticoagulation prior to admission four remained alive and one died. The prevalence of patients with lupus anticoagulant did not show any difference between the two study groups. The Kaplan Meier method showed that lupus anticoagulant was not associated with a higher mortality (log-rank test:0.4265; $p=0.4294$ ) (Fig. 1) When the combined outcome of death or need for mechanical ventilation in survivors was taken into account, the difference in the prevalence of patients with lupus anticoagulant between the patients with the combined outcome $(n=76)$ and survivors who did not require mechanical ventilation $(n=116)$ was not significant $(52.6 \%$ versus $47.4 \%$; $\mathrm{p}=0.4806$ ). A multivariate analysis with the primary outcome and with the combined outcome as the dependent variable was exploited. The following variables were tested and dichotomized before the analysis: age $(<65$ or $\geq 65$ years), history of hypertension (yes or not), history of lung disease (yes or not), BMI ( $<30$ or $\geq 30$ ), eGFR $(<60$ or $\geq 60 \mathrm{ml} / \mathrm{min})$, C-reactive protein $(<100$ or $\geq 100 \mathrm{mg} / \mathrm{L}$ ), d-dimer ( $<200$ or $\geq 200 \mathrm{ng} / \mathrm{ml}$ ), high-sensitivity troponin: $<20$ or $\geq 20 \mathrm{pg} / \mathrm{ml}$ ), Oxygen Saturation ( $\leq 93$ or $>93 \%$ ), Lactase dehydrogenase ( $<300$ or $\geq 300 \mathrm{U} / \mathrm{L})$. Table 2 summarizes the predictors of the primary and combined outcome. Predictors of death were older age, obesity, presence of hypertension, low Oxygen Saturation and high troponin on admission. Predictors of death or need for mechanical ventilation in survivors were obesity, Oxygen Saturation and high troponin on admission.

Table 3 reports the patients stratified by presence/absence of lupus anticoagulant; as shown, patients with lupus anticoagulant were older, and had BMI, C-reactive protein, highsensitivity troponin, prothrombin time and activated partialthromboplastin time significantly higher than patients without lupus anticoagulant. None of the five patients on therapeutic anticoagulation prior to admission had lupus anticoagulant. The multivariate analysis showed that predictors of the presence of lupus anticoagulant were age > 65 years (OR 2.2194;

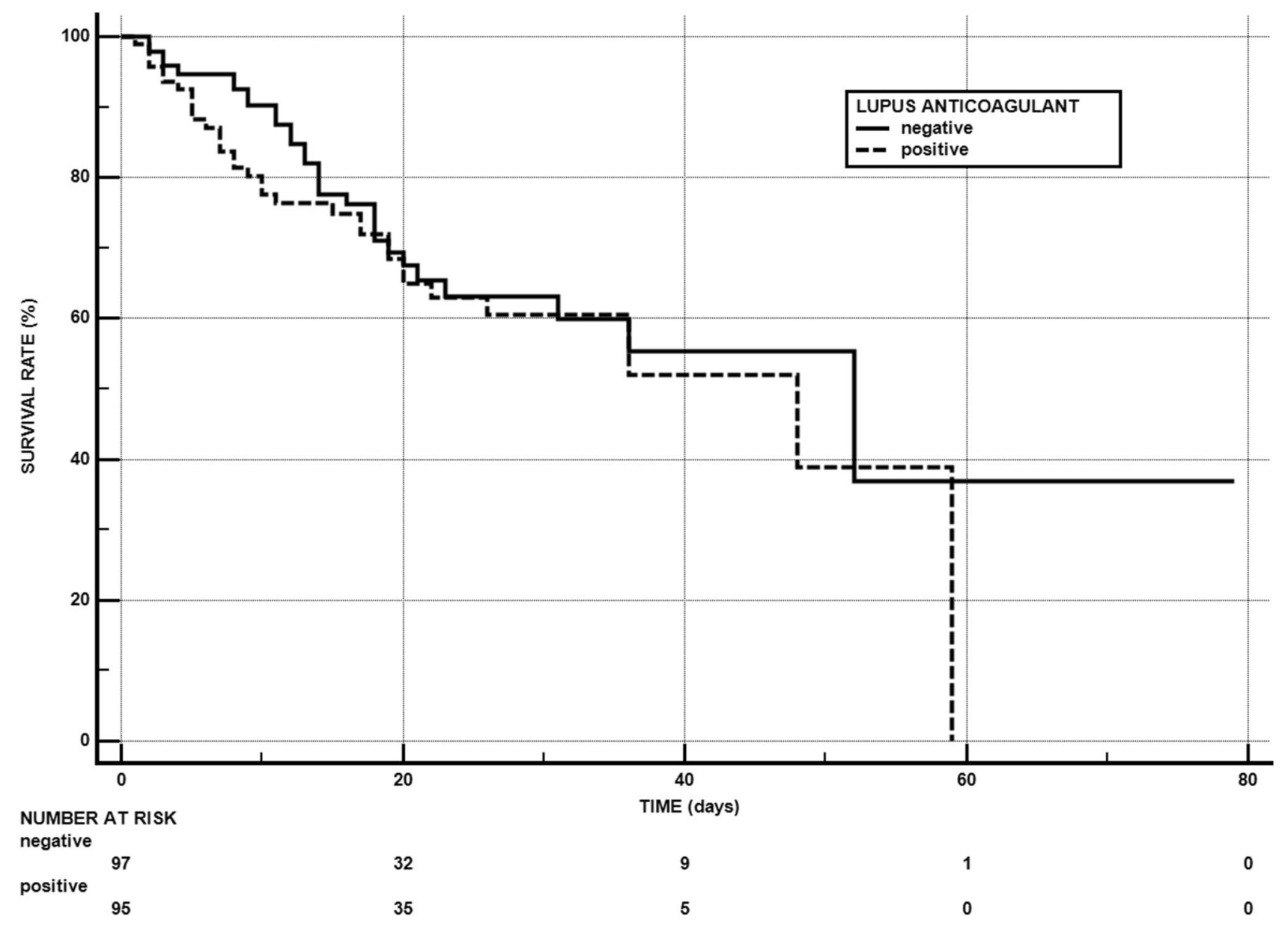

Fig. 1 Kaplan-Meier survival curve according to presence/absence of lupus anticoagulant in patients hospitalized for COVID-19 during the follow-up period (18.8 \pm 13.2 days-range $1-79)$. For each time inter-

val, survival probability is calculated as the number of subjects surviving divided by the number of patients at risk ("number at risk"). Subjects who have died are not counted as "at risk" 
Table 1 Features of the whole population and of survivors and non-survivors

\begin{tabular}{|c|c|c|c|c|c|}
\hline Variable & Reference range & $\begin{array}{l}\text { Total patients } \\
(\mathrm{n}=192)\end{array}$ & $\begin{array}{l}\text { Survivors } \\
(\mathrm{n}=130)\end{array}$ & $\begin{array}{l}\text { Non-survivors } \\
(\mathrm{n}=62)\end{array}$ & p-value \\
\hline Age (years) & & $69.4 \pm 14.5$ & $66.6 \pm 14.8$ & $75.2 \pm 12.1$ & 0.0001 \\
\hline Males (\%) & & 58.3 & 56.9 & 61.3 & 0.5670 \\
\hline History of Diabetes (\%) & & 17.2 & 17.7 & 16.1 & 0.7889 \\
\hline History of Hypertension (\%) & & 45.3 & 35.4 & 50.0 & 0.0540 \\
\hline History of CVD (\%) & & 23.4 & 23.1 & 24.2 & 0.8647 \\
\hline History of Lung Disease (\%) & & 9.9 & 6.9 & 16.1 & 0.0463 \\
\hline BMI & & $27.9 \pm 4.8$ & $26.4 \pm 3.8$ & $30.9 \pm 5.3$ & $<0.0001$ \\
\hline eGFR (ml/min) & $>90$ & $71.3 \pm 27.1$ & $74.2 \pm 25.0$ & $65.2 \pm 30.5$ & 0.0541 \\
\hline $\mathrm{CRP}(\mathrm{mg} / \mathrm{L})$ & $<5$ & $142.2 \pm 118.0$ & $118.3 \pm 88.7$ & $176.6 \pm 117.7$ & 0.0342 \\
\hline D-dimer (ng/ml) & $<200$ & $1762.2 \pm 5189.6$ & $1049.0 \pm 3097.2$ & $3255.7 \pm 7790.6$ & $<0.0001$ \\
\hline High-sensitivity Troponin (pg/ml) & $<20$ & $40.0 \pm 88.3$ & $14.8 \pm 18.7$ & $92.6 \pm 139.6$ & $<0.0001$ \\
\hline Lupus anticoagulant $(\%)$ & Negative & 49.5 & 47.7 & 53.2 & 0.4745 \\
\hline Prothrombin time (s) & $9.9-12.9$ & $13.6 \pm 3.0$ & $13.4 \pm 2.9$ & $14.0 \pm 3.1$ & 0.1904 \\
\hline Activated partial-thromboplastin time (s) & $25-45$ & $32.0 \pm 5.2$ & $32.2 \pm 5.4$ & $31.7 \pm 4.9$ & 0.5470 \\
\hline $\mathrm{SaO} 2(\%)$ & $>93$ & $89.9 \pm 7.4$ & $91.6 \pm 5.8$ & $86.6 \pm 9.2$ & $<0.0001$ \\
\hline Lactate dehydrogenase (U/L) & $125-300$ & $371.7 \pm 229.6$ & $336.0 \pm 146.3$ & $446.5 \pm 333.5$ & 0.0082 \\
\hline Therapeutic anticoagulation (\%) & & 49.0 & 34.6 & 79.0 & $<0.0001$ \\
\hline
\end{tabular}

$C V D$ cardiovascular disease, $B M I$ body mass index, $e G F R$ estimated glomerular filtration rate, $C R P$ C-reactive protein, $\mathrm{SaO} 2$ oxygen saturation

Table 2 Predictors of outcomes in patients hospitalized for COVID-19

\begin{tabular}{lllllr}
\hline Predictors & $\begin{array}{l}\text { Regression } \\
\text { coefficient } \beta\end{array}$ & Standard error SE & Odds Ratio & 95\%CI & p-value \\
\hline Primary outcome (death) & & & & & \\
Age & 1.1293 & 0.4006 & 3.0935 & $1.4107-6.7839$ & 0.0048 \\
BMI & 1.2871 & 0.2693 & 3.6222 & $2.1368-6.1401$ & $<0.0001$ \\
History of hypertension & -0.6616 & 0.2749 & 0.5160 & $0.2999-0.8879$ & 0.0169 \\
SaO2 & 1.1088 & 0.3676 & 3.0309 & $1.4746-6.2296$ & 0.0026 \\
High-sensitivity troponin & 0.6539 & 0.2903 & 1.9230 & $1.0887-3.3967$ & 0.0243 \\
Combined outcome (death or need for mechanical ventilation in survivors) & & \\
BMI & 0.9808 & 0.2499 & 2.6666 & $1.6338-4.3522$ & 0.0001 \\
SaO2 & 0.8571 & 0.2962 & 2.3563 & $1.3184-4.2112$ & 0.0038 \\
High-sensitivity troponin & 0.6073 & 0.2419 & 1.8355 & $1.1426-2.9486$ & 0.0120 \\
\hline
\end{tabular}

Primary outcome. Variables not included into the model: d-dimer, eGFR, lactate dehydrogenase, history of a lung disease and C-reactive protein

Combined outcome. Variables not included into the model: age, d-dimer, history of hypertension, eGFR, lactate dehydrogenase, history of a lung disease and C-reactive protein

95\% CI 95\% confidence interval, $B M I$ body mass index, $\mathrm{SaO} 2$ oxygen saturation, $e G F R$ estimated glomerular filtration rate
95\%CI 1.1839-4.1606; $\mathrm{p}=0.0129)$ and prolonged prothrombin time (OR 2.5329; 95\% CI 1.3906-4.6137; $\mathrm{p}=0.0024)$.

\section{Discussion}

Two recent studies found conflicting results about the association between lupus anticoagulant and thromboembolic complications of COVID-19 [13, 20]. Indeed, the study by
Siguret did not show any significant difference in the prevalence of lupus anticoagulant between critically ill COVID-19 patients with and those without thrombotic complications [13], while the study by Reyes Gil documented a significantly higher percentage of thrombotic events in patients with than in those without lupus anticoagulant [20]. These contrasting results may be due to differences in age, severity of disease, interference due to drugs used for anticoagulation, and setting $[13,20]$. Both studies documented a high 
Table 3 Features and outcomes of the patients stratified by presence/absence of lupus anticoagulant

\begin{tabular}{|c|c|c|c|c|c|}
\hline Variable & Reference range & $\begin{array}{l}\text { Total patients } \\
(\mathrm{n}=192)\end{array}$ & $\begin{array}{l}\text { Patients with positive } \\
\text { lupus anticoagulant } \\
(\mathrm{n}=95)\end{array}$ & $\begin{array}{l}\text { Patients with negative } \\
\text { lupus anticoagulant } \\
(\mathrm{n}=97)\end{array}$ & p-value \\
\hline Age (years) & & $69.4 \pm 14.5$ & $73.0 \pm 14.0$ & $65.8 \pm 14.1$ & 0.0005 \\
\hline Males (\%) & & 58.3 & 63.2 & 53.6 & 0.1808 \\
\hline History of Diabetes (\%) & & 17.2 & 17.9 & 16.5 & 0.7976 \\
\hline History of Hypertension (\%) & & 45.3 & 47.4 & 43.3 & 0.5722 \\
\hline History of CVD (\%) & & 23.4 & 26.3 & 20.6 & 0.3527 \\
\hline History of Lung Disease (\%) & & 9.9 & 11.6 & 8.2 & 0.4408 \\
\hline BMI & & $27.9 \pm 4.8$ & $28.7 \pm 5.6$ & $27.0 \pm 3.7$ & 0.0136 \\
\hline eGFR (ml/min) & $>90$ & $71.3 \pm 27.1$ & $68.1 \pm 28.5$ & $74.4 \pm 25.6$ & 0.1096 \\
\hline $\mathrm{CRP}(\mathrm{mg} / \mathrm{L})$ & $<5$ & $142.2 \pm 118.0$ & $151.6 \pm 101.5$ & $123.0 \pm 101.7$ & 0.0072 \\
\hline D-dimer (ng/ml) & $<200$ & $1762.2 \pm 5189.6$ & $1568.9 \pm 3700.7$ & $1950.3 \pm 6332.7$ & 0.4407 \\
\hline High-sensitivity Troponin (pg/ml) & $<20$ & $40.0 \pm 88.3$ & $50.1 \pm 105.3$ & $29.9 \pm 66.5$ & 0.0009 \\
\hline Lupus anticoagulant (\%) & Negative & 49.5 & 100 & 0 & 1.000 \\
\hline Prothrombin time (s) & $9.9-12.9$ & $13.6 \pm 3.0$ & $14.5 \pm 3.7$ & $12.8 \pm 1.8$ & $<0.0001$ \\
\hline Activated partial-thromboplastin time (s) & $25-45$ & $32.0 \pm 5.2$ & $34.2 \pm 5.7$ & $29.9 \pm 3.7$ & $<0.0001$ \\
\hline $\mathrm{SaO} 2(\%)$ & $>93$ & $89.9 \pm 7.4$ & $89.6 \pm 8.1$ & $90.3 \pm 6.7$ & 0.5028 \\
\hline Lactate dehydrogenase (U/L) & $125-300$ & $371.7 \pm 229.6$ & $356.7 \pm 166.6$ & $386.4 \pm 278.0$ & 0.2752 \\
\hline Therapeutic anticoagulation (\%) & & 49.00 & 53.7 & 44.3 & 0.1960 \\
\hline Non-survivors $(\%)$ & & 32.3 & 34.7 & 29.9 & 0.4745 \\
\hline $\begin{array}{l}\text { Non survivors and survivors who required } \\
\text { mechanical ventilation }(\%)\end{array}$ & & 39.6 & 42.1 & 37.1 & 0.4806 \\
\hline
\end{tabular}

$C V D$ cardiovascular disease, $B M I$ body mass index, $C R P$ : $e G F R$ estimated glomerular filtration rate, $C R P$ C-reactive protein, $S a O 2$ oxygen saturation

prevalence of lupus anticoagulant as well as several other studies [14, 17-19]. Tang suggested that the interference of anticoagulation may explain the high prevalence of the lupus anticoagulant found in patients with COVID-19 [22]. Some authors believe that false positive lupus anticoagulant testing may be expected when there is a marked elevation of C-reactive protein $[19,22]$; therefore, lupus anticoagulant may be transient in patients with COVID-19 and devoid of any predictive power for thromboembolism. The present study confirms a high prevalence of lupus anticoagulant among patients hospitalized for COVID-19, and first shows that the presence of lupus coagulant does not predict either mortality or the need for mechanical ventilation in these patients. It is of interest to understand the reasons for our findings. In our study lupus anticoagulant was evaluated on admission and before anticoagulation and therefore the interference due to anticoagulation was not present. The presence of lupus anticoagulant may be due to several clinical conditions, such as infections, inflammation, autoimmunity [23] that can be present in patients with COVID-19. Indeed, in addition to viral infection, hyperinflammation is common in COVID-19 and can cause of a worse clinical course [1]. Therefore, the degree of inflammation may affect the presence of lupus anticoagulant. We found that patients with lupus anticoagulant had C-reactive protein significantly higher than patients without lupus anticoagulant, even if this association was not confirmed in multivariate analysis. Autoimmune mechanisms may also be associated to the presence of lupus anticoagulant [23]. Really, we first found that there is a high prevalence of antinuclear antibodies and other auto-antibodies in patients with COVID-19 [14]. Unfortunately, in the present study we did not evaluate autoimmune markers other than lupus anticoagulant. Therefore, we cannot exclude that other auto-immune mechanisms can have a role in the presence of lupus anticoagulant.

However, the above hypothesis may explain the high prevalence of lupus anticoagulant, but not the lack of predictive power of lupus anticoagulant for the prognosis. It is well-known that antiphospholipid autoantibodies (anticardiolipin and anti-beta2-glycoprotein) are used together with lupus anticoagulant for the diagnosis of the antiphospholipid syndrome [11,23]. A positivity for anticardiolipin, anti-beta2-glycoprotein and lupus anticoagulant in the same subject (triple positivity) is usually associated with a very high thrombotic risk, while a double positivity (presence of anticardiolipin and anti-beta2-glycoprotein, but absence of lupus anticoagulant) is characterized by a lower risk $[11,23]$. It is unclear the degree of thrombotic risk associated with the presence of lupus anticoagulant alone [11,23]. Considering that anticardiolipin 
and anti-beta2-glycoprotein were not tested in the present study, we cannot exclude that a small group with a triple positivity may have a worse prognosis. However, several studies found not only a very low presence of antiphospholipid antibodies in patients with COVID-19 but also the lack of association between these antibodies and the prognosis of COVID-19 [7, 10-16, 20].The lack of association between lupus anticoagulant and a worse prognosis in our study may also be due to the fact that the presence of lupus anticoagulant may be transient in patients with COVID-19 and therefore it may have a limited and temporary pathophysiological effect. Unfortunately, we evaluated lupus anticoagulant on admission but not during the follow-up. This is a study limitation. Another explanation for our finding may be represented by the fact that anticoagulation may be effective in reducing the potential thrombotic risk linked to lupus anticoagulant. Indeed, all our patients were early treated with prophylactic or therapeutic anticoagulation. In particular, all the patients were treated with a prophylactic dose of enoxaparin on admission. Five patients were on therapeutic anticoagulation prior to admission and were continued on it during hospitalization. Patients on prophylactic anticoagulation were started on therapeutic anticoagulation as soon as a thrombotic complication was suspected or there was a worsening disease progression. The proportion of patients treated with therapeutic anticoagulation was significantly higher among non-survivors than survivors. This is not surprising, as patients who were started on therapeutic anticoagulation had more severe and complicated forms of disease and thus a higher risk of death. Although the proportion of patients on therapeutic anticoagulation was greater in patients with lupus anticoagulant when compared to those without lupus anticoagulant, the difference does not attain statistical significance. However, we recorded a high mortality. This may suggest that the thrombotic risk remained high because of other mechanisms non-responsive to anticoagulation with heparin, such as the acute decrease in antithrombin [9] or other autoimmune mechanisms [14]. In addition, there are studies in critically ill patients without lupus anticoagulant and antiphospholipid antibodies but with a lot of other coagulation abnormalities that can greatly increase the thrombotic risk [5, 6, 8, 9, 24]. However, future work should provide further information about a comprehensive and effective assessment of the individual thrombotic risk in COVID-19, as recent autopsy findings showed in situ thrombosis in all the patients died because of COVID-19 and the involvement of the renin angiotensin system, as SARS-Cov2 uses the Angiotensin-Converting Enzyme 2 (ACE2) receptor for host cell entry and ACE2 expression seems to correlate with susceptibility to SARS-CoV infection $[3,25]$.
It may be of interest to know how many thrombotic events occurred in our study; unfortunately, we are not able to provide the exact number, as complications were not adequately documented in some patients. This is a study limitation.

Interestingly, our study confirms the major role of obesity, low oxygen saturation and high troponin levels in predicting mortality and need for a mechanical ventilation, as recently outlined [26, 27]. These variables can be used as useful prognostic markers together with other cardiac and coagulation parameters $[5,25]$.

Taken together our study shows that the presence of lupus anticoagulant is common in patients hospitalized for COVID-19, but it does not seem to be a prognostic marker for mortality and need for mechanical ventilation. The reasons for these findings remain unclear. If our study results are confirmed, lupus anticoagulant in COVID-19 should be considered as a side-effect of a higher inflammatory state or of other mechanisms rather than a causing effect for thromboembolism. However, our data confirm the major role of obesity, low oxygen saturation and elevated troponin levels as predictors of a worse prognosis in these patients.

Authors' contributions CG, AC and PG contributed to concept, design and supervision of the study, interpretation of data and writing the manuscript. CG and AC performed statistical analysis. GM, CR, LM, $\mathrm{ET}, \mathrm{MC}, \mathrm{VS}, \mathrm{LB}$, TA contributed to the acquisition and interpretation of data and critical revision of the manuscript.

Funding Current Research of Clinical Institute Beato Matteo, Vigevano Italy.

\section{Compliance with ethical standards}

Conflict of interest None of the authors has conflicts of interest.

\section{References}

1. Gazzaruso C, Carlo Stella N, Mariani G (2020) Impact of antirheumatic drugs and steroids on clinical course and prognosis of COVID-19. Clin Rheumatol 39(8):2475-2477. https://doi. org/10.1007/s10067-020-05239-5

2. Bikdeli B, Madhavan MV, Jimenez D et al (2020) COVID-19 and thrombotic or thromboembolic disease: implications for prevention, antithrombotic therapy, and follow-up. J Am Coll Cardiol. https://doi.org/10.1016/j.jacc.2020.04.031

3. Connors JM, Levy JH (2020) COVID-19 and its implications for thrombosis and anticoagulation. Blood. https://doi.org/10.1182/ blood.2020006000

4. Wichmann D, Sperhake JP, Lütgehetmann M et al (2020) Autopsy findings and venous thromboembolism in patients with COVID19. Ann Intern Med. https://doi.org/10.7326/M20-2003

5. Tang N, Li D, Wang X, Sun Z (2020) Abnormal coagulation parameters are associated with poor prognosis in patients with novel coronavirus pneumonia. J Thromb Haemost 18(4):844-847. doi:https://doi.org/10.1111/jth.14768 
6. Han H, Yang L, Liu R, Liu F, Wu KL, Li J, Liu XH, Zhu CL (2020) Prominent changes in blood coagulation of patients with SARS-CoV-2 infection. Clin Chem Lab Med 25(7):1116-1120. https://doi.org/10.1515/cclm-2020-0188

7. Zhang Y, Cao W, Jiang W (2020) Profile of natural anticoagulant, coagulant factor and anti-phospholipid antibody in critically ill COVID-19 patients. J Thromb Thrombolysis 9:1-7. doi:https:// doi.org/10.1007/s11239-020-02182-9

8. Nougier C, Benoit R, Simon M et al (2020) Hypofibrinolytic state and high thrombin generation may play a major role in sars-cov2 associated thrombosis. J Thromb Haemost. https://doi. org $/ 10.1111 /$ jth. 15016

9. Gazzaruso C, Paolozzi E, Valenti C et al (2020) Association between antithrombin and mortality in patients with COVID-19. A possible link with obesity. Nutr Metab Cardiovasc Dis 2020, https://doi.org/10.1016/j.numecd.2020.07.040

10. Zhang Y, Xiao M, Zhang S et al (2020) Coagulopathy and antiphospholipid antibodies in patients with Covid-19. N Engl J Med 382(17):e38. https://doi.org/10.1056/NEJMc2007575

11. Devreese KMJ, Linskens EA, Benoit D, Peperstraete H (2020) Antiphospholipid antibodies in patients with COVID-19: a relevant observation? J Thromb Haemost. https://doi.org/10.1111/ jth.14994

12. Gatto M, Perricone C, Tonello M et al (2020) Frequency and clinical correlates of antiphospholipid antibodies arising in patients with SARS-CoV-2 infection: findings from a multicentre study on 122 cases. Clin Exp Rheumatol 38(4):754-759

13. Siguret V, Voicu S, Neuwirth M, Delrue M, Gayat E, Stépanian A, Mégarbane B (2020) Are antiphospholipid antibodies associated with thrombotic complications in critically ill COVID-19 patients? Thromb Res 195:74-76. doi:https://doi.org/10.1016/j. thromres.2020.07.016

14. Gazzaruso C, Carlo Stella N, Mariani G et al (2020) High prevalence of antinuclear antibodies and lupus anticoagulant in patients hospitalized for SARS-CoV2 pneumonia. Clin Rheumatol 39(7):2095-2097. doi:https://doi.org/10.1007/s10067-020-05180 $-7$

15. Harzallah I, Debliquis A, Drénou B (2020) Lupus anticoagulant is frequent in patients with Covid-19: Response to Reply. J Thromb Haemost. https://doi.org/10.1111/jth.14980

16. Galeano-Valle F, Oblitas CM, Ferreiro-Mazón MM, AlonsoMuñoz J, Del Toro-Cervera J, di Natale M, Demelo-Rodríguez $P$ (2020) Antiphospholipid antibodies are not elevated in patients with severe COVID-19 pneumonia and venous thromboembolism. Thromb Res 192:113-115. doi:https://doi.org/10.1016/j.throm res.2020.05.017

17. Harzallah I, Debliquis A, Drénou B (2020) Lupus anticoagulant is frequent in patients with Covid-19. J Thromb Haemost. https:// doi.org/10.1111/jth.14867
18. Bowles L, Platton S, Yartey N et al (2020) Lupus anticoagulant and abnormal coagulation tests in patients with Covid-19. N Engl J Med 16(3):288-290. https://doi.org/10.1056/NEJMc2013656

19. Helms J, Tacquard C, Severac F, CRICS TRIGGERSEP Group (Clinical Research in Intensive Care and Sepsis Trial Group for Global Evaluation and Research in Sepsis), et al (2020) High risk of thrombosis in patients with severe SARS-CoV-2 infection: a multicenter prospective cohort study. Intensive Care Med 46(6):1089-1098. https://doi.org/10.1007/s00134-020-06062-х

20. Reyes Gil M, Barouqa M, Szymanski J, Gonzalez-Lugo JD, Rahman S, Billett HH (2020) J Assessment of lupus anticoagulant positivity in patients with coronavirus disease 2019 (COVID-19) JAMA Netw Open. 3(8):e2017539

21. Pengo V, Tripodi A, Reber G,et al (2009) Update of the guidelines for lupus anticoagulant detection. Subcommittee on Lupus Anticoagulant/Antiphospholipid Antibody of the Scientific and Standardisation Committee of the International Society on Thrombosis and Haemostasis. J Thromb Haemost 7(10):1737-1740. doi:https ://doi.org/10.1111/j.1538-7836.2009.03555.x

22. Tang N (2020) Response to "Lupus anticoagulant is frequent in patients with Covid-19" (JTH-2020-00483). J Thromb Haemost. https://doi.org/10.1111/jth.14890

23. Cervera R (2017) Antiphospholipid syndrome. Thromb Res 151(suppl 1):S43-S47

24. Adam EH, Zacharowski K, Miesbach W (2020) A comprehensive assessment of the coagulation profile in critically ill COVID-19 patients. Thromb Res 194:42-44

25. Eketunde AO, Mellacheruvu SP, Oreoluwa P (2020) A review of postmortem findings in patients with COVID-19. Cureus 12(7):e9438

26. Rottoli M, Bernante P, Belvedere A (2020) How important is obesity as a risk factor for respiratory failure, intensive care admission and death in hospitalised COVID-19 patients? Results from a single Italian centre. Eur J Endocrinol. https://doi.org/10.1530/ EJE-20-0541

27. Qin JJ, Cheng X, Zhou F et al (2020) Redefining cardiac biomarkers in predicting mortality of inpatients with COVID-19. Hypertension. https://doi.org/10.1161/HYPERTENSIONAHA .120 .15528

Publisher's Note Springer Nature remains neutral with regard to jurisdictional claims in published maps and institutional affiliations. 